\title{
Mobilization of Medial and Lateral Frontal-Striatal Circuits in Cocaine Users and Controls: An Interleaved TMS/BOLD Functional Connectivity Study
}

\author{
Colleen A Hanlon ${ }^{*, 1,2,3}$, Logan T Dowdle', Hunter Moss', Melanie Canterberry' and Mark S George ${ }^{1,2,3,4}$ \\ 'Department of Psychiatry and Behavioral Sciences, Medical University of South Carolina, Charleston, SC, USA; '2Department of Neurosciences, \\ Medical University of South Carolina, Charleston, SC, USA; ${ }^{3}$ Center for Biomedical Imaging, Medical University of South Carolina, Charleston, \\ SC, USA; ${ }^{4}$ Ralph H. Johnson VA Medical Center, Charleston, SC, USA
}

\begin{abstract}
The integrity of frontal-striatal circuits is an area of great interest in substance dependence literature, particularly as the field begins to develop neural circuit-specific brain stimulation treatments for these individuals. Prior research indicates that frontal-striatal connectivity is disrupted in chronic cocaine users in a baseline (resting) state. It is unclear, however, if this is also true when these circuits are mobilized by an external source. In this study, we measured the functional and structural integrity of frontal-striatal circuitry involved in limbic arousal and executive control in 36 individuals - 18 cocaine-dependent individuals with a history of failed quit attempts and 18 age-matched controls. This was achieved by applying a transcranial magnetic stimulation to the medial prefrontal cortex (Brodmann area 10) and the dorsolateral prefrontal cortex (lateral Brodmann 9) while participants rested in the MRI scanner (TMS/BOLD imaging). Relative to the controls, cocaine users had a lower ventral striatal BOLD response to MPFC stimulation. The dorsal striatal BOLD response to DLPFC stimulation however was not significantly different between the groups. Among controls, DLPFC stimulation led to a reciprocal attenuation of MPFC activity (BA 10), but this pattern did not exist in cocaine users. No relationship was found between regional diffusion metrics and functional activity. Considered together these data suggest that, when engaged, cocaine users can mobilize their executive control system similar to controls, but that the 'set point' for mobilizing their limbic arousal system has been elevated — an interpretation consistent with opponent process theories of addiction.
\end{abstract}

Neuropsychopharmacology (20 I6) 4I, 3032-304I; doi:I0.1038/npp.20 I6.I I4; published online 27 July 2016

\section{INTRODUCTION}

Chronic cocaine use is among the most difficult substance use disorders to treat. High relapse rates are likely due to a combination of factors that involve heightened limbic drive toward drug-related cues and difficulty controlling their urges to use the drug. From a neuroimaging perspective, limbic drive and executive control are regulated by distinct, complementary frontal-striatal neural circuits in the brain (Alexander et al, 1986; Middleton and Strick, 2002; Ongur and Price, 2000). The limbic loop includes projections from the medial prefrontal cortex (MPFC) to the ventral striatum. The executive control loop includes projections from the dorsolateral prefrontal cortex (DLPFC) to the dorsal striatum. Cocaine users typically have an attenuated prefrontal cortex response to tasks that require mobilization

* Correspondence: Dr CA Hanlon, Departments of Psychiatry and Neurosciences, Center for Advanced Imaging Research, Medical University of South Carolina, Charleston, SC 29425, USA, Tel: + I 843 792-5732, Fax: + I 843 792-7457, E-mail: hanlon@musc.edu

Received 24 November 2015; revised 17 May 2016; accepted 18 May 2016; accepted article preview online 4 July 2016 of both the limbic circuit (eg natural rewards) (Asensio et al, 2010a; Cheetham et al, 2010; Kelley and Berridge, 2002). One of the only things which reliably activates the MPFC in this population appears to be drug cues (Childress et al, 2008; Goldstein et al, 2007; Hester and Garavan, 2009; Prisciandaro et al, 2013).

While task-based neuroimaging studies that probe frontalstriatal circuitry involved in arousal to non-drug cues typically reveal 'hypofrontality' among cocaine users (Asensio et al, 2010a), recent resting state neuroimaging studies investigating these limbic circuits have revealed higher resting state functional connectivity in mesolimbic frontal-striatal circuits relative to controls (Camchong et al, 2011; Hu et al, 2015) (Note: lower connectivity between anterior cingulate-striatal circuit has also been observed $(\mathrm{Hu}$ et al, 2015)). There are several possible interpretations to the apparent gap in the results of task-based neuroimaging and resting state neuroimaging studies. One interpretation is that low task evoked baseline activity in the prefrontal cortex may be related to human subject's factors (eg required concentration and task engagement), which are not as critical for resting state studies. Another possibility, however, is that the basic neural architecture is intact (ie resting state 
connectivity), but the threshold for engagement of these arousal/salience circuits is disrupted-a hypothesis consistent with the opponent process theory of addiction (Koob, 1996; Koob and Le Moal, 2008a, b).

The primary goal of this study was to quantify frontalstriatal functional connectivity in a cohort of cocaine users and matched controls that were resting in an MRI scanner as their MPFC and DLPFC were externally activated. The data that emerge from this experiment may provide a neurobiological basis for the emerging data that a potentiating form of repetitive transcranial magnetic stimulation (TMS) to the DLPFC ((Camprodon et al, 2007; Politi et al, 2008; Terraneo et al, 2016), or an attenuating form of TMS to the MPFC (Hanlon et al, 2015) appears to dampen craving among cocaine users. To minimize the impact of the methodological and human subject's factors, we employed an experimental technique that activates the prefrontal cortex (like many task-based studies) without requiring the individual to concentrate on a task. To probe the integrity of these circuits, we applied a series of single pulses of TMS to the MPFC and the DLPFC in the MRI scanner. This interleaved TMS/BOLD imaging technique leads to a momentary elevation of BOLD signal in the cortex directly affected by the induced electrical field (MPFC and DLPFC), as well as areas monosynaptically connected to the site (Baudewig et al, 2001; Bestmann et al, 2003; Bestmann et al, 2005; Bohning et al, 1999; Bohning et al, 2000a; Bohning et al, 1998; Bohning et al, 2000b). Although this technique does not likely reflect how a circuit is organically activated when engaged in a task, it is an informative hybrid between pure resting state and pure task-based imaging. Prior studies by our group have demonstrated that it is possible to differentially activate the dorsal and ventral striatum through this technique (Hanlon et al, 2013). We tested the opposing hypotheses that, among cocaine users, externally evoked DLPFC and MPFC activity would lead to: (1) attenuated activity in the corresponding frontal-striatal circuits (as taskbased studies would predict) or (2) amplified activity in these circuits (as resting state studies would predict) relative to age, gender, and education-matched controls.

\section{MATERIALS AND METHODS}

\section{Subjects}

In this experiment we were primarily interested in investing frontal-striatal circuitry in current cocaine users rather than individuals engaged in treatment. This selection strategy was motivated by an interest in eventually developing brain stimulation treatment paradigms for individuals not otherwise choosing to enroll in traditional outpatient programs. Consequently, non-treatment seeking chronic cocaine users and age and gender-matched non-drug using controls were recruited from the Charleston, SC metropolitan area via newspaper ads, broadcast messages, and word-or-mouth. Twenty cocaine users and 20 non-drug using controls signed informed consent and completed the study, which was approved by the Medical University of South Carolina Institutional Review Board. They then provided urine samples to test for current illicit drug use and were administered the DSM-IV-based Mini International Neuropsychiatric Interview (Sheehan et al, 1998), the Beck
Table I Demographic and Cocaine Use History for Participants

\begin{tabular}{|c|c|c|c|c|}
\hline \multirow[b]{2}{*}{ Age (years) } & \multicolumn{2}{|c|}{ Controls } & \multicolumn{2}{|c|}{ Users } \\
\hline & 35.1 & $(7.8)$ & 36.2 & $(8.6)$ \\
\hline Females & $43 \%$ & & $56 \%$ & \\
\hline Alcohol use $\mathrm{a}^{\mathrm{a}}$ & 4.1 & $(1.9)$ & 5.8 & $(4.2)$ \\
\hline Daily nicotine use & $22 \%$ & & $56 \%$ & \\
\hline Nicotine dependence ${ }^{b}$ & 1.0 & $(0)$ & 1.7 & $(1.0)$ \\
\hline Depressive symptoms ${ }^{c}$ & 2.0 & $(2.46)$ & 4.7 & $(5.0)$ \\
\hline Anxiety state ${ }^{d}$ & 26.4 & $(10.0)$ & 30.25 & $(\mid 1.7)$ \\
\hline Anxiety trait ${ }^{\mathrm{d}}$ & 26.6 & $(9.5)$ & 33.5 & $(\mid 1.6)$ \\
\hline Age of first cocaine use & - & - & 19.6 & $(3.9)$ \\
\hline Total years of use & - & - & 16.6 & $(4.6)$ \\
\hline Years at current level & - & - & 8.9 & $(6.7)$ \\
\hline
\end{tabular}

Values are expressed as means $( \pm \mathrm{SD})$.

${ }^{a}$ Alcohol Use Disorders Identification test (AUDIT-C).

bagerstrom Test of Nicotine Dependence.

'Beck Depression Inventory.

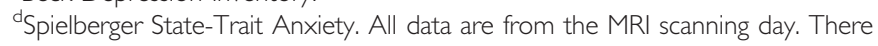
were no significant differences between groups on any of these parameters $(p>0.05)$.

Depression Inventory (Beck et al, 1996), and the Alcohol Use Disorders Identification Test (AUDIT) (Babor et al, 2001). To be eligible, the cocaine users had to meet DSM-IV criteria for cocaine dependence, have a positive urine drug screen for cocaine (indicating use within $\sim 72 \mathrm{~h}$ ), and could not meet criteria for dependence on any other class of drugs. Exclusionary criteria for both groups included past week use of illegal psychoactive drugs (other than cocaine for the cocaine group), current use of prescription medication, a score of more than 15 on the AUDIT, smoking $>1$ pack of cigarettes per day, a Fagerstrom Test of Nicotine Dependence score $>3$, and a lifetime history of head injury with loss of consciousness. While there were more daily smokers in the cocaine group (10/18) than the control group (4/18), the average AUDIT score and Fagerstrom Test for Nicotine Dependence score were not significantly different. The demographic and drug use information for these individuals is included in Table 1. The final sample size was 36 as data from 2 cocaine users and 2 controls were not included in the final analysis due to excessive movement artifact in the functional MRI images ( $>3 \mathrm{~mm}$ in any direction over the course of a run) (see Preprocessing). These 36 individuals are the participants described in Table 1 and in all remaining sections of this manuscript.

\section{Motor Threshold}

On the day of the MRI scan, the individuals were asked to abstain from cocaine use for $12 \mathrm{~h}$ prior to the visit. No individuals demonstrated clinical signs that they were actively high upon arrival to the MRI center. Participants were brought to the imaging center where resting motor threshold (RMT) was determined. TMS was applied using a Magstim SuperRapid stimulator, which generates biphasic electrical pulses $(250 \mu \mathrm{s})$. The stimulator was located outside of the scanning room. The pulses were delivered through a $8 \mathrm{~m}$ cable that first attached to the bottom of an RF filter, and 
then passed through the waveguide into the MR scanning room where it was led through the bore of the MRI and terminated in a custom nonferromagnetic figure-of-eight TMS coil (Bohning et al, 2003; George et al, 2003; Li et al, 2004; Nahas et al, 2001).

\section{TMS-BOLD Image Acquisition}

This study was performed on a Siemens 3T TIM trio scanner (Siemens, Erlangen, Germany). Following the motor threshold procedure, participants were positioned supine on the scanner bed and the TMS coil was mounted in the MR head coil (RAPID Biomedical (Rimpar, Germany) 12 channel head array ) with a custom TMS coil holder adjustable in six directions (X, Y, Z, pitch, yaw, and roll) (Bohning et al, 2003). While participants lay supine on the bed, the position of the TMS coil was aligned to the location of F3 (Position 1: left DLPFC) during one functional MRI run and to FP1 (Position 2: MPFC) during the other functional MRI run. A fiducial was affixed to the center of the TMS coil for offline verification of position. Participants then received two interleaved TMS-BOLD imaging runs: (1) coil positioned over F3, (2) coil positioned over FP1. This order was randomized. During each run, a series of 12 single pulses of TMS were applied to either the MPFC or the DLPFC, with a $12 \mathrm{~s}$ interpulse interval. These pulses were modeled as events in the linear regression. Specifically, biphasic TMS pulses $(250 \mu \mathrm{s})$ were applied at $110 \%$ of RMT during a $100 \mathrm{~ms}$ TR delay following every fourth $\operatorname{TR}(4,8,12 \ldots)$. After the first functional run, the bed of the MRI scanner was retracted from the bore such that the TMS coil could be moved to the second position. Low-resolution $\mathrm{T}_{1}$-weighted anatomical images were acquired again for alignment and offline verification of coil position, before commencing the second TMS-BOLD imaging run.

\section{Anatomical Image Acquisition}

High-resolution $\mathrm{T}_{1}$-weighted structural images were obtained by using a magnetization-prepared rapid acquisition of gradient echo sequence (160 slices, $50 \%$ distance factor, $256 \times 256$ matrix, TR $=1900 \mathrm{~ms}, \mathrm{TE}=4.18 \mathrm{~ms}$, flip angle $=9^{\circ}$, slice thickness $=1.0 \mathrm{~mm}$, and GRAPPA). Diffusion-weighted images were obtained by using a twice-refocused echo-planar sequence with two diffusion weightings $\left(b=0,1000 \mathrm{~s} / \mathrm{mm}^{2}\right)$ along 30 diffusion-encoding directions (50 slices, $0 \%$ distance factor, $222 \times 222$ field of view, $74 \times 74$ matrix, $\mathrm{TR}=6700 \mathrm{~ms}$, $\mathrm{TE}=87 \mathrm{~ms}$, slice thickness $=3 \mathrm{~mm}$, partial Fourier encoding: $6 / 8$, no interpolation, and 2 averages).

\section{Scalp-Cortex Distance Quantification}

Given that the effects of TMS on cortical depolarization are proportional to the distance between the skull and the cortex (Kozel et al, 2000; Stokes et al, 2005), we calculated the distance from the scalp to the cortex on the transverse plane on MPRAGE images of each individual (Mango ver. 3.7; Research Imaging Institute, UTHSA, Lancaster \& Martinez 2005) (Supplementary Figure S1). The average distance from the participant-specific placement of FP1 to the nearest cortex (controls: $13.5 \mathrm{~mm} \pm 2.8$; users: $14.2 \mathrm{~mm} \pm 3.3$ ) and $\mathrm{F} 3$ to the nearest cortex (controls: $14.5 \mathrm{~mm} \pm 1.7$; users:
$15.1 \mathrm{~mm} \pm 3.1)$ was not significantly different between the groups. Of note, while some of the cocaine users had noticeable cortical atrophy, easily visualized as sulcal enlargement, this did not appear to have a significant effect on the distance between the gyri and the skull (See Supplementary Figure S2 for an example). All analyses were done with these distances as covariates (demonstrated in Supplementary Figure S3).

\section{Functional MRI Data Analysis Preprocessing}

Spatial preprocessing was performed with standard parametric mapping techniques (SPM12, London, UK) in MATLAB 7.14 (Mathworks, Natick, MA). Images were first converted from DICOM format to 4D NIfTI files, and motion corrected (Realign: 6 parameter, rigid body realignment to first image in each time series using a least squares approach). Normalization parameters, bias correction, and anatomical tissue maps were determined simultaneously, using the Segment toolbox. Individual anatomical images were stripped of their skulls by masking the bias-corrected image with the combined tissue masks of gray matter, white matter, and CSF. The functional images derived from realignment were coregistered, through the mean image, to the skull-stripped anatomical image (Coregister: Estimate, using normalized mutual information). Coregistered images were then normalized (Normalize: Write) to MNI template space with the nonlinear warps derived from the Segment tool. Functional images were masked (to remove the skull) and smoothed ( $8 \mathrm{~mm}$ FWHM Gaussian kernel) prior to any between-group analyses. The motion was limited to $3 \mathrm{~mm}$ and residual movement parameters (X, Y, Z, pitch, yaw, and roll) were included as regressors on first-level within-subject analyses. Individuals that exceeded this motion limit on either of the functional runs were excluded from the analysis. Note: of the four individuals who were excluded from the analysis based on $>3 \mathrm{~mm}$ motion, the excessive movement occurred in the F3/DLPFC stimulation run in all four participants.

\section{Voxel-Based Linear Regression-TMS Pulse-Based Functional Connectivity}

For this analysis first-level contrast maps were developed for each participant. This was done by specifying first-level models for TMS onsets in both the DLPFC and MPFC condition. The TMS pulses in each condition were modeled as instantaneous events, which were then convolved with the canonical hemodynamic response function (double gamma, as provided by SPM12). High-pass filtering was set such that fluctuations with a period $>128 \mathrm{~s}$ would be removed to account for signal drift within the scanner. Contrasts were generated for each participant's neural response (as measured by BOLD) to DLPFC and MPFC stimulation. A full factorial analysis (TMS Location $\times$ Group) was then done using these parameter estimates (contrast maps) (see Supplementary Figure S3 for design matrix). This design matrix was used to develop all statistical contrasts and subsequent Tables and Figures contained in this manuscript. Main effects, interactions, and post hoc t-tests were then created for all relevant contrasts. All analyses were performed with statistical correction at both the voxel and cluster level $(p<0.05$, family-wise error-corrected clusters). The analysis was restricted to an inclusive mask of the left and right frontal 
Table 2 Healthy Controls_-Brain Regions Significantly Modulated by TMS to the MPFC/Frontal Pole and the DLPFC

\begin{tabular}{|c|c|c|c|c|c|c|c|c|}
\hline & & \multirow[t]{2}{*}{ Significant clusters ${ }^{\mathbf{a}}$} & \multirow[t]{2}{*}{$\mathbf{B A}^{\mathbf{b}}$} & \multicolumn{3}{|c|}{ MNI coordinates } & \multirow[t]{2}{*}{$\operatorname{Max}^{c}$} & \multirow[t]{2}{*}{ Cluster level } \\
\hline & & & & $x$ & $y$ & $\mathbf{z}$ & & \\
\hline \multicolumn{9}{|l|}{ MPFC stimulation: elevated BOLD signal } \\
\hline \multirow[t]{2}{*}{ I cluster, $k=2877$} & $L$ & Caudate, parahipp., insula, amygdala & 34 & -18 & 5 & -18 & 4.52 & 0.002 \\
\hline & L\&R & Caudate, orbital PFC, insula, amygdala & 11,47 & 27 & | | & -16 & 4.30 & \\
\hline MPFC stimulation: attenuated BOLD signal & & No significant clusters & & & & & & \\
\hline \multicolumn{9}{|l|}{ DLPFC stimulation: elevated BOLD signal } \\
\hline I cluster, $k=670$ & $\mathrm{R}$ & Putamen, caudate, amygdala & & 21 & 11 & -13 & 6.30 & $<0.000$ \\
\hline \multicolumn{9}{|l|}{ DLPFC stimulation: attenuated BOLD signal } \\
\hline \multirow[t]{3}{*}{ I cluster, $k=540$} & $L$ & Medial PFC & 10 & -6 & 68 & ।I & 3.87 & 0.003 \\
\hline & $L$ & Middle and inferior frontal gyrus & 44 & -48 & 20 & 44 & 3.42 & \\
\hline & $L$ & Frontal pole & 45 & -39 & 56 & 17 & 3.42 & \\
\hline
\end{tabular}

Clusters of brain regions that had a significant increase or decrease in BOLD signal following a series of TMS pulses applied to the left medial prefrontal cortex/frontal pole (MPFC; EEG coordinate FPI based in International I0-20 system) and to the left dorsolateral prefrontal cortex (DLPFC; EEG coordinate F3). Stereotactic coordinates from the Montreal Neurologic Institute $(\mathrm{MNI})$ template are listed for each cluster of voxels that exceeded family-wise error correction $(p<0.05)$. The coordinates for the top three local maxima in each cluster are listed along with their anatomical labels (Harvard-Oxford Atlas), and the uncorrected $p$-values for each cluster. Monte Carlo simulations used to determine family-wise error correction level.

aLocal maxima determined from probabilistic Harvard-Oxford Atlas implemented in FSL.

${ }^{\mathrm{b}}$ Brodmann area.

${ }^{\mathrm{C}} \mathrm{T}$ value at the voxel level.

cortex and subcortical projection regions including the caudate, putamen, pallidum, thalamus, insula, amygdala, and hippocampus (AAL atlas implemented in WFU_Pick Atlas). The minimum number of voxels required to reflect adjustment for family-wise error was determined via AlphaSim (with 1000 Monte Carlo simulations). Smoothness estimation was calculated on the positive effect map obtained in second level analysis $(\mathrm{rmm}=3$ based on the equation: $1.412<\mathrm{L}<1.732$ wherein $\mathrm{L}$ is the voxel dimensions in the largest plane after resampling ( $3 \mathrm{~mm})$ (REST toolbox ver 1.8).

\section{Diffusion Tensor Imaging Data}

A full description of the diffusion tensor imaging data analysis strategy and results can be found in Supplementary Material. As this experiment was not explicitly designed to make comparisons of fractional anisotropy values between cocaine users and controls, it is not sufficiently powered to address that question. The primary goal of acquiring the diffusion data was to determine if there was a relationship between fractional anisotropy and TMS-evoked BOLD response in the frontal-striatal circuits of interest. Tractbased spatial statistics revealed that there were no significant differences in regional anisotropy between the groups nor did FA values in the regions of interest surrounding the cortical target areas (FP1 and F3) nor the striatum correlate with the TMS-evoked BOLD changes.

\section{RESULTS}

\section{Limbic Network Connectivity (MPFC (FP1) Stimulation): Matched Controls}

In controls, TMS applied to the left MPFC (FP1) led to significant increases in the BOLD signal in two clusters, which primarily included the left ventral caudate (ventral striatum), parahippocampal gyrus, insula and amygdala (Cluster 1), and the anterior cingulate cortex and left frontal pole (Cluster 2). There were no regions with significant decreases following MPFC stimulation (Table 2, Figure 1a).

\section{Limbic Network Connectivity (MPFC (FP1) Stimulation): Cocaine Users}

Unlike the controls, TMS applied to the left MPFC (FP1) of cocaine users did not lead to a significant increase in any brain region. It did, however, lead to a significant decrease in the superior frontal gyrus (Table 3, Figure 1b). 


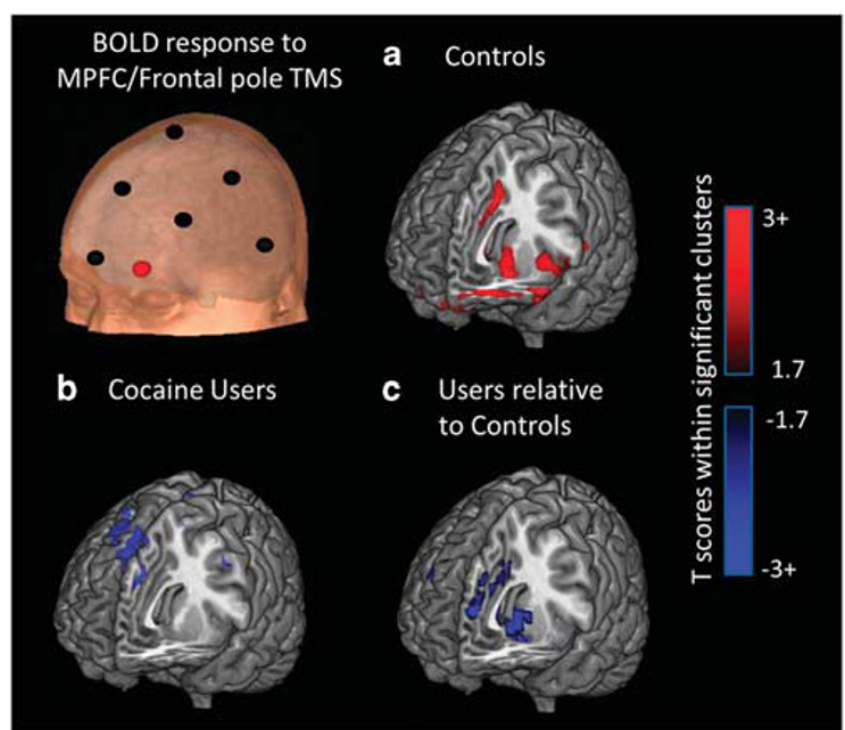

Figure I The BOLD response to medial prefrontal cortex (MPFC) stimulation. A series of single pulses of transcranial magnetic stimulation were applied to the left MPFC/frontal pole of healthy controls and cocaine users while they rested in an MRI scanner. The coil was positioned over the FPI location (red dot) of each individual based on the EEG International 10-20 system (black dots show standard positions). The brain regions that had a significantly elevated BOLD signal (red colormap) and significantly attenuated BOLD signal (blue colormap) are displayed for the controls (a) and the cocaine users (b), and the cocaine users relative to the controls (c). The statistical maps for all of these comparisons arose from the same full factorial design (SPMI2; Family-wise error-corrected clusters $p<0.05)$. Only voxels that fell within those significant clusters are displayed.

\section{Limbic Network Connectivity: Between Groups}

Relative to the controls, the cocaine users had significantly lower BOLD signal in two clusters: the left and right caudate (ventral striatum), putamen (dorsal striatum), accumbens (ventral striatum), and amygdala (Cluster 1) frontal pole (Cluster 2). (Supplementary Table S1, Figure 1c). There were no regions in which the cocaine users had a greater response to $\mathrm{MPFC} /$ frontal pole stimulation.

\section{Executive Network Connectivity (DLPFC (F3) Stimulation): Matched Controls}

In controls, TMS applied to the left DLPFC (F3) led to significant increases in the BOLD signal in two clusters, which primarily included the left putamen (dorsal striatum), caudate, insula and amygdala (Cluster 1), and the anterior cingulate cortex (Cluster 2). There was also a significant decrease in BOLD signal in one cluster following DLPFC stimulation that included the MPFC, inferior frontal gyrus, and the frontal pole (Table 2, Figure 2a).

\section{Executive Network Connectivity (DLPFC (F3) Stimulation): Cocaine Users}

Similar to the controls, TMS applied to the left DLPFC (F3) led to significant increases in the BOLD signal in two clusters, which primarily included the putamen (dorsal striatum), caudate and thalamus (Cluster 1), and the anterior

Table 3 Chronic Cocaine Users_-Brain Regions Significantly Modulated by TMS to the MPFC/Frontal Pole and the DLPFC

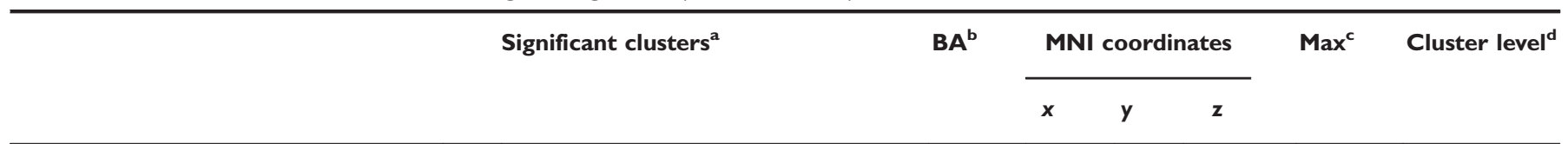

MPFC stimulation: elevated BOLD signal

No significant clusters

MPFC stimulation: attenuated BOLD signal

I cluster, $k=58$ |

\section{R Superior frontal gyrus}

L Superior frontal gyrus

$\begin{array}{rrrrr}8 & 3 & 41 & 50 & 4.39 \\ 9 & -4 & 44 & 35 & 3.49\end{array}$

3.49
0.009

\begin{tabular}{|c|c|c|c|c|c|c|c|}
\hline \multirow[t]{2}{*}{ I cluster, $k=708$} & $\mathrm{R}$ & Putamen & & 18 & 8 & -13 & 5.42 \\
\hline & $\mathrm{L}$ & Caudate, Thalamus & & -6 & 5 & 2 & 4.65 \\
\hline \multirow[t]{2}{*}{ I cluster, $k=1026$} & $\mathrm{~L}$ & Anterior cingulate, Superior frontal gyrus & 32,24 & -3 & 38 & -4 & 4.60 \\
\hline & $\mathrm{R}$ & Anterior cingulate & 32,24 & 6 & 38 & 5 & 4.38 \\
\hline
\end{tabular}

DLPFC stimulation: elevated BOLD signal

Clusters of brain regions that had a significant increase or decrease in BOLD signal following a series of TMS pulses applied to the left medial prefrontal cortex/frontal pole (MPFC; EEG coordinate FPI based in International 10-20 system) and to the left dorsolateral prefrontal cortex (DLPFC; EEG coordinate F3). Stereotactic coordinates from the Montreal Neurologic Institute (MNI) template are listed for each cluster of voxels that exceeded family-wise error correction ( $p<0.05)$. The coordinates for the local maxima in each cluster are listed along with their anatomical labels (Harvard-Oxford Atlas), and the uncorrected $p$-values for each cluster.

aLocal maxima determined from probabilistic Harvard-Oxford Atlas implemented in FSL.

brodmann area.

${ }^{\mathrm{c}} \mathrm{T}$ value at the voxel level.

¿Uncorrected $p$-value listed. $K=$ the number of voxels in the cluster. All clusters reach family-wise error correction $p \leq 0.05$ (AlphaSim, Monte Carlo simulations). 


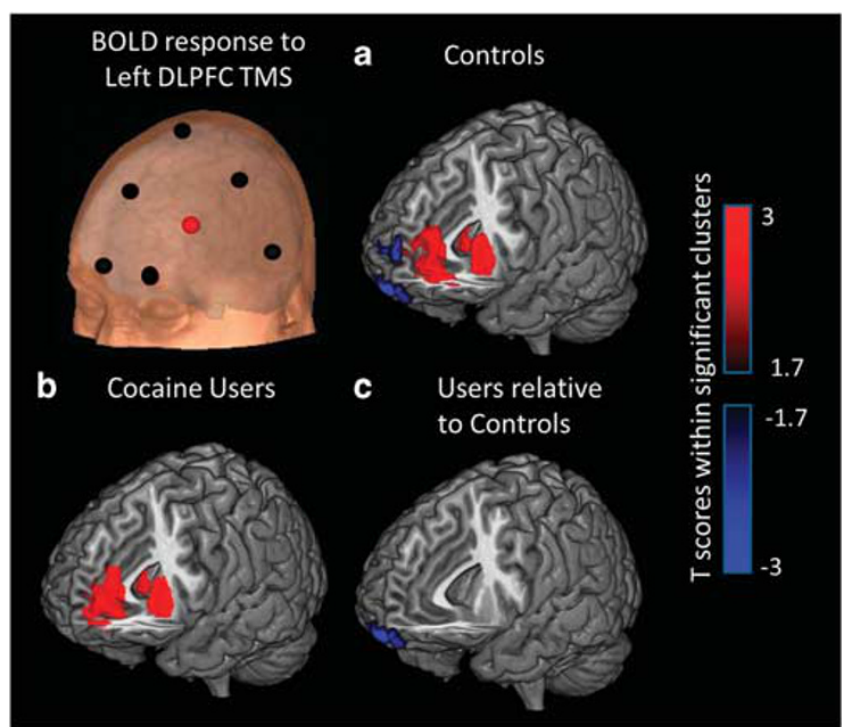

Figure 2 The BOLD response to dorsolateral prefrontal cortex (DLPFC) stimulation. A series of single pulses of transcranial magnetic stimulation were applied to the left DLPFC of healthy controls and cocaine users while they rested in an MRI scanner. The coil was positioned over the F3 location (red dot) of each individual based on the EEG International 10-20 system (black dots show standard positions). The brain regions that had a significantly elevated BOLD signal (red colormap) and significantly attenuated BOLD signal (blue colormap) are displayed for the controls (a), the cocaine users (b), and the cocaine users relative to the controls (c). The statistical maps for all of these comparisons arose from the same full factorial design (SPMI 2; Family-wise error-corrected clusters $p<0.05)$. Only voxels that fell within those significant clusters are displayed.

cingulate cortex (Cluster 2). There was no significant decrease in BOLD signal (Table 3, Figure 2b).

\section{Executive Network Connectivity: Between Groups}

Relative to the controls, there was only one cluster in which the cocaine users had significantly lower BOLD signal. The cluster was centered in the frontal pole/medial orbital gyrus. There were no regions in which the cocaine users had a greater response to DLPFC/frontal pole stimulation. There was also no difference in the dorsal striatal response to DLPFC stimulation between the groups (Supplementary Table S2, Figure 2c).

\section{Association Between BOLD Response to TMS and Clinical Variables in Cocaine Users}

There was no correlation between the BOLD response to TMS stimulation and state of trait anxiety, depressive symptoms, alcohol use severity, age of first use of cocaine, or total years of cocaine use.

\section{DISCUSSION}

The functional integrity of both medial and lateral frontalstriatal circuits is an important area of discovery in addiction research, as these circuits govern limbic arousal and cognitive control- dual and opponent processes implicated in the evolution of drug dependence, abstinence, and often relapse (Koob, 1996; Koob and Le Moal, 2008a). While the functional connectivity of these circuits in cocainedependent individuals has been measured in a resting state (Gu et al, 2010; Hu et al, 2015; Li et al, 2000; Tomasi et al, 2010), the goal of this study was to investigate the functional connectivity in these circuits when they were engaged by an external source. The primary results demonstrate that (1) ventral striatal areas responsible for limbic arousal are not as responsive to the same level of medial prefrontal stimulation in cocaine users as controls (consistent with opponent process theory), (2) dorsal striatal areas typically implicated in cognitive control and habit formation have similar response profiles in cocaine users and controls, and (3) the reciprocal relationship between DLPFC stimulation and Brodmann 10 (MPFC) attenuation observed in controls is disrupted in cocaine users. Considered in the context of other studies that have demonstrated high resting state connectivity in these frontal-striatal networks, the present data suggests that while the basic scaffolding of the frontalstriatal circuits may be present in cocaine users, stimulation of Brodmann 10 (by an external source) does not evoke the typical increase in striatal BOLD signal, which is observed in controls. Taken together, these data provide us with some insights regarding the development of new circuit-specific treatment strategies for these individuals, such as TMS.

\section{Reciprocal Connectivity Between the Medial Prefrontal Cortex and the Dorsolateral Prefrontal Cortex}

One of the most interesting observations of the present study is the reciprocal relationship between activity in the DLPFC and the MPFC in the controls and in the cocaine users. Consistent with previous studies, a series of single pulses of TMS to the DLPFC resulted in an increase in dorsal striatal BOLD signal in both controls (Hanlon et al, 2013) and cocaine users. This is aligned with PET imaging studies demonstrating that repetitive TMS to the left DLPFC leads to a reduction in $[(11) C]$ raclopride binding in the ipsilateral caudate, suggesting elevated dopamine release (Strafella et al, 2001). Interestingly, however, in the present study DLPFC stimulation also led to a reciprocal decrease in medial orbitofrontal (BA 10) BOLD signal in the controls, but not the cocaine users. The reciprocal relationship between the DLPFC and BA 10 in controls is consistent with other studies, which have revealed negative connectivity between the central executive network and the salience network (Chen and Etkin, 2013a; Liston et al, 2014). It is also consistent with PET studies showing that $10 \mathrm{~Hz}$ rTMS to the left DLPFC effects dopamine release in the medial orbitofrontal cortex and anterior cingulate cortex, as revealed by [(11)C]FLB 457 binding, a ligand sensitive to D2 receptors (Cho and Strafella, 2009). Interestingly, although this was not the primary aim of their study, Cho et al 2015 administered $10 \mathrm{~Hz}$ rTMS to the dorsal MPFC with a double cone coil (which has deeper cortical penetration than a flat coil) and observed a change in dopamine displacement in the dorsal striatum, but not the ventral striatum, an elegant demonstration of the well-established topography of dorsal and ventral frontal-striatal circuits (Haber and Knutson, 2010). Although these explanations will require future experiments, to our knowledge, this is the first study to directly investigate the positive and the negative relationships between these two 
prefrontal cortical areas in controls and substance-dependent individuals.

From one perspective, it may seem obvious that the MPFC BOLD signal should decrease when the DLPFC is engaged by TMS given that the MPFC is a node in the default mode network (Damoiseaux et al, 2006), and this has been empirically observed in rTMS studies of depression in the past (Liston et al, 2014). It is not obvious, however, why DLPFC stimulation would lead to a decrease in BA 10 of the controls but this would not be present in cocaine users. One possibility is that cocaine users have a lower response to DLPFC stimulation in general. Another possibility is that Brodmann 10 in cocaine users is structurally compromised (Franklin et al, 2002; Hanlon et al, 2011; Matochik et al, 2003). Both of these explanations, however, are weakened by the observation that DLPFC stimulation leads to an increase in dorsal striatum BOLD in both groups. In addition, MPFC stimulation in the cocaine users leads to reciprocal inhibition of the DLPFC.

Several other explanations for the lack of this reciprocal attenuation in the cocaine users may be: (1) a functional disconnection between these two complementary frontalstriatal networks (Liang et al, 2015), or (2) attenuated BOLD signal dynamics within the MPFC of cocaine users. Yet another interpretation is that Brodmann 10 is not as responsive to the typical cascade of signals from the DLPFC, which lead to an attenuated BOLD response. Unfortunately, the structural connectivity between the MPFC and DLPFC in primates is presently not well understood and there is still considerable uncertainty regarding the biophysics of a negative BOLD signal. Although this study was not designed to answer those questions specifically, these results illuminate an important difference in the reciprocal connectivity between executive and limbic circuitry within cocaine users.

\section{Ventral Medial Prefrontal Cortex: Impaired Mobilization in Cocaine Users}

One of the primary goals of this study was to investigate the impact of frontal pole/ventral MPFC stimulation on ventral striatal activity. This was motivated by the knowledge that cocaine users appear to have a very low neural response to natural rewards (Cheetham et al, 2010), which typically engage the limbic system (eg the ventral MPFC, ACC, and striatum) (Asensio et al, 2010a; Kelley and Berridge, 2002). In addition, several resting state functional MRI studies have demonstrated lower baseline resting state functional connectivity with the MPFC in cocaine users (Gu et al, 2010; Gu et al, 2007; Tomasi et al, 2010), though other studies have demonstrated that cocaine users have higher connectivity in these circuits (Camchong et al, 2011; Hu et al, 2015). The present study demonstrated that left frontal pole stimulation in the controls led to an increase in BOLD signal in a network of limbic regions including the caudate, parahippocampal gyrus, and the amygdala. In the cocaine users, however, there were no brain regions amplified by left frontal pole stimulation. A possible explanation is that Brodmann 10 is less responsive to an equivalent stimulus in the cocaine users relative to the controls. This would suggest that the 'set point' for Brodmann 10 activity may be shifted in cocaine users-a philosophy consistent with opponent process theory (Koob and Le Moal, 2008a, b) and one that could

explain its involvement in cue-induced craving tasks despite low activity to natural rewards.

\section{Implications for Treatment Development-Dorsal Medial and Lateral PFC Brain Stimulation}

One of the biggest areas of growth in addiction research at the moment is neural circuit-specific treatment modalities. Preclinical studies, eg, have demonstrated that optogenetic modulation of these mesolimbic and mesocortical circuits can modulate drug-taking behavior in a causal manner (Chen et al, 2013c; Stefanik et al, 2013). Clinical studies are now investigating repetitive TMS (rTMS) as a tool to change drug- craving and drug-taking behavior. RTMS can induce long-term potentiation (LTP) or long-term depression (LTD) depending on the frequency chosen. Most of the rTMS studies to date have applied an LTP-inducing form of TMS to the DLPFC in an effort to decrease craving ((Bellamoli et al, 2014; Gorelick et al, 2014; Hanlon et al, 2015). The efficacy of TMS to the DLPFC as a tool to decrease craving in cocaine users was first demonstrated by Camprodon and collegues (2007) and recently explored in a large, multi-week trial in Italy (Terraneo et al, 2016). It is not immediately obvious why increasing activity in the DLPFC (an element in the executive control network) would decrease craving (a function typically ascribed to the ventral medial PFC and ventral subcortical areas). The data from the present study, however, demonstrate that there is a reciprocal relationship between DLPFC stimulation and subsequent attenuation of Brodmann 10 in the MPFC (an area involved in craving). This result, which is consistent with similar studies in individuals with mood disorders (Chen et al, 2013b; Liston et al, 2014), and it provides a biological mechanism through which DLPFC may be effective at attenuating craving.

In addition, this study demonstrates that relationship between the stimulation of the DLPFC (by an external source) and elevated activity in the dorsal striatum (regions involved in planning and habit formation) is intact among cocaine users. This is encouraging data for clinicians and treatment providers, as this neural circuitry is typically engaged by cognitive behavioral therapy. It is possible that LTP-like repetitive TMS to the DLPFC may be well suited as an adjuvant to cognitive behavioral therapy among substance-dependent individuals.

\section{Implications for Treatment Development-Ventral Medial PFC Stimulation}

While most of the TMS literature in psychiatry presently has focused on stimulating the DLPFC, there is growing interest in applying TMS to the dorsal medial (Bakker et al, 2015) and ventral medial (Hanlon et al, 2015) prefrontal cortex. A recent sham-controlled neuroimaging study by our group demonstrated that 3600 pulses of continuous theta burst stimulation (an LTD-like form of TMS) to the left frontal pole/MPFC/BA 10 decreased stimulus-evoked activity in the ventral MPFC and the nucleus accumbens (Hanlon et al, 2015), brain regions typically invoked by drug cues and implicated in cue-primed relapse. The present study demonstrates that, relative to controls, the amplitude of TMS required to evoke a significant BOLD signal in striatal limbic circuitry may be relatively high in the cocaine users. 
However, given that this circuit is more disrupted in cocaine users developing a treatment, which perhaps could restore its 'set point' to a typical level may be a fruitful treatment direction. These data are particularly interesting in the context of longitudinal neuroimaging findings from (Camchong et al 2014), who demonstrated that functional connectivity between the left frontal pole (the area stimulated in the present experiment) and the nucleus accumbens, as well as the left frontal pole and the cingulate cortex were directly related to an individual's propensity to relapse to cocaine. The MPFC response to non-drug rewards also appears to be modulated by striatal dopamine (Asensio et al, 2010b), suggesting that modulating this circuit might restore arousal to non-drug rewards. Although resting state functional MRI does not necessarily generalize to the stimulus-evoked BOLD signal studies in the present experiment, both of these approaches point toward the frontal pole as an important candidate structure for sustained abstinence from cocaine.

The primary limitation of this study is the relatively small sample of community-recruited cocaine users who had long cocaine use histories and were also regular tobacco smokers. While the nicotine dependence scores were not significantly different between the smokers in the cocaine group and the control group, there were twice as many daily smokers in the cocaine group (56\%) than the control group (22\%). The high nicotine smoking rates in this sample is typical for community-based cohorts of cocaine users, however, the relatively small sample precludes a robust assessment of the relative contribution of each of these drugs to the biological phenomena we observed. Consequently, these data should likely only be interpreted in the framework of a typical sample of cocaine users in the community. In addition none of these individuals were actively seeking treatment for dependence at the time of enrollment. In summary, this experiment demonstrates that frontal-striatal circuitry involved in limbic arousal is significantly harder to mobilize in cocaine users than controls, but the frontal-striatal circuitry involved in executive control is largely intact. Both of these observations have implications for shaping future brain stimulation treatment in cocaine users.

The development of rTMS as a tool to aid in the treatment of substance use disorders is, however, truly in its infancy. The field still needs many more active sham-controlled, double-blinded trials to help clinicians and scientists determine the most effective frequencies, cortical locations, and substance abuse populations that are good candidates for this intervention. As the field moves forward, we hope that the results of this manuscript will provide a platform for future biologically based treatment development for cocainedependent individuals, as well as other populations struggling with an imbalance between limbic drive and executive control.

\section{FUNDING AND DISCLOSURE}

The authors declare no conflict of interest.

\section{ACKNOWLEDGMENTS}

We would like to thank William DeVries for assisting in participant screening, Jayce Doose for engineering support,
James Purl for MRI acquisition support, Dr Truman S. Brown for data quality assessment and interleaved TMS/ BOLD imaging sequence optimization, and Scott Henderson for editorial support. This work was supported by R01DA0036617, T32DA007288, R25DA020537, and UL1 TR001450. Dr Hanlon's work has been funded exclusively by the National Institutes of Health. MSG has received funding from the National Institutes of Health, the Department of Defense, and the Veterans Administration. He has also served as a consultant to several major TMS device companies including MagStim, Neuronetics, Magventure, and Brainsway.

\section{REFERENCES}

Alexander GE, DeLong MR, Strick PL (1986). Parallel organization of functionally segregated circuits linking basal ganglia and cortex. Annu Rev Neurosci 9: 357-381.

Asensio S, Romero MJ, Palau C, Sanchez A, Senabre I, Morales JL et al (2010a). Altered neural response of the appetitive emotional system in cocaine addiction: an fMRI Study. Addict Biol 15: 504-516.

Asensio S, Romero MJ, Romero FJ, Wong C, Alia-Klein N, Tomasi D et al (2010b). Striatal dopamine D2 receptor availability predicts the thalamic and medial prefrontal responses to reward in cocaine abusers three years later. Synapse 64: 397-402.

Babor TF, Higgins-Biddle JC, Saunders JB, Monteiro MG (2001). The Alcohol Use Disorders Identification Test (AUDIT) Manual. World Health Organization: Geneva, Switzerland.

Bakker N, Shahab S, Giacobbe P, Blumberger DM, Daskalakis ZJ, Kennedy SH et al (2015). rTMS of the dorsomedial prefrontal cortex for major depression: safety, tolerability, effectiveness, and outcome predictors for $10 \mathrm{~Hz}$ versus intermittent theta-burst stimulation. Brain Stimul 8: 208-215.

Baudewig J, Siebner HR, Bestmann S, Tergau F, Tings T, Paulus W et al (2001). Functional MRI of cortical activations induced by transcranial magnetic stimulation (TMS). Neuroreport 12: 3543-3548.

Beck AT, Steer RA, Brown GK (1996). Manual for the Beck Depression Inventory-II. Psychological Corporation: San Antonio, TX.

Bellamoli E, Manganotti P, Schwartz RP, Rimondo C, Gomma M, Serpelloni G (2014). rTMS in the treatment of drug addiction: an update about human studies. Behav Neurol 2014: 815215.

Bestmann S, Baudewig J, Frahm J (2003). On the synchronization of transcranial magnetic stimulation and functional echo-planar imaging. J Magn Reson Imaging 17: 309-316.

Bestmann S, Baudewig J, Siebner HR, Rothwell JC, Frahm J (2005). BOLD MRI responses to repetitive TMS over human dorsal premotor cortex. Neuroimage 28: 22-29.

Bohning DE, Denslow S, Bohning PA, Walker JA, George MS (2003). A TMS coil positioning/holding system for MR imageguided TMS interleaved with fMRI. Clin Neurophysiol 114: 2210-2219.

Bohning DE, Shastri A, McConnell KA, Nahas Z, Lorberbaum JP, Roberts DR et al (1999). A combined TMS/fMRI study of intensity-dependent TMS over motor cortex. Biol Psychiatry 45: 385-394.

Bohning DE, Shastri A, McGavin L, McConnell KA, Nahas Z, Lorberbaum JP et al (2000a). Motor cortex brain activity induced by $1-\mathrm{Hz}$ transcranial magnetic stimulation is similar in location and level to that for volitional movement. Invest Radiol 35: 676-683.

Bohning DE, Shastri A, Nahas Z, Lorberbaum JP, Andersen SW, Dannels WR et al (1998). Echoplanar BOLD fMRI of brain 
activation induced by concurrent transcranial magnetic stimulation. Invest Radiol 33: 336-340.

Bohning DE, Shastri A, Wassermann EM, Ziemann U, Lorberbaum JP, Nahas $\mathrm{Z}$ et al (2000b). BOLD-f MRI response to single-pulse transcranial magnetic stimulation (TMS). J Magn Reson Imaging 11: $569-574$.

Camchong J, Macdonald AW 3rd, Mueller BA, Nelson B, Specker S, Slaymaker V et al (2014). Changes in resting functional connectivity during abstinence in stimulant use disorder: a preliminary comparison of relapsers and abstainers. Drug Alcohol Depend 139: $145-151$.

Camchong J, MacDonald AW 3rd, Nelson B, Bell C, Mueller BA, Specker S et al (2011). Frontal hyperconnectivity related to discounting and reversal learning in cocaine subjects. Biol Psychiatry 69: 1117-1123.

Camprodon JA, Martinez-Raga J, Alonso-Alonso M, Shih MC, Pascual-Leone A (2007). One session of high frequency repetitive transcranial magnetic stimulation (rTMS) to the right prefrontal cortex transiently reduces cocaine craving. Drug Alcohol Depend 86: 91-94.

Cheetham A, Allen NB, Yucel M, Lubman DI (2010). The role of affective dysregulation in drug addiction. Clin Psychol Rev 30: 621-634.

Chen AC, Etkin A (2013a). Hippocampal network connectivity and activation differentiates post-traumatic stress disorder from generalized anxiety disorder. Neuropsychopharmacology 38: 1889-1898.

Chen AC, Oathes DJ, Chang C, Bradley T, Zhou ZW, Williams LM et al (2013b). Causal interactions between fronto-parietal central executive and default-mode networks in humans. Proc Natl Acad Sci USA 110: 19944-19949.

Chen BT, Yau HJ, Hatch C, Kusumoto-Yoshida I, Cho SL, Hopf FW et al (2013c). Rescuing cocaine-induced prefrontal cortex hypoactivity prevents compulsive cocaine seeking. Nature 496: 359-362.

Childress AR, Ehrman RN, Wang Z, Li Y, Sciortino N, Hakun J et al (2008). Prelude to passion: limbic activation by "unseen" drug and sexual cues. PLoS One 3: e1506.

Cho SS, Koshimori Y, Aminian K, Obeso I, Rusjan P, Lang AE et al (2015). Investing in the future: stimulation of the medial prefrontal cortex reduces discounting of delayed rewards. Neuropsychopharmacology 40: 546-553.

Cho SS, Strafella AP (2009). rTMS of the left dorsolateral prefrontal cortex modulates dopamine release in the ipsilateral anterior cingulate cortex and orbitofrontal cortex. PLoS One 4: e6725.

Damoiseaux JS, Rombouts SA, Barkhof F, Scheltens P, Stam CJ, Smith SM et al (2006). Consistent resting-state networks across healthy subjects. Proc Natl Acad Sci USA 103: 13848-13853.

Franklin TR, Acton PD, Maldjian JA, Gray JD, Croft JR, Dackis CA et al (2002). Decreased gray matter concentration in the insular, orbitofrontal, cingulate, and temporal cortices of cocaine patients. Biol Psychiatry 51: 134-142.

George MS, Nahas Z, Kozol FA, Li X, Yamanaka K, Mishory A et al (2003). Mechanisms and the current state of transcranial magnetic stimulation. CNS Spectr 8: 496-514.

Goldstein RZ, Tomasi D, Rajaram S, Cottone LA, Zhang L, Maloney $\mathrm{T}$ et al (2007). Role of the anterior cingulate and medial orbitofrontal cortex in processing drug cues in cocaine addiction. Neuroscience 144: 1153-1159.

Gorelick DA, Zangen A, George MS (2014). Transcranial magnetic stimulation in the treatment of substance addiction. Ann NY Acad Sci 1327: 79-93.

Gu H, Salmeron BJ, Ross TJ, Geng X, Zhan W, Stein EA et al (2010). Mesocorticolimbic circuits are impaired in chronic cocaine users as demonstrated by resting-state functional connectivity. Neuroimage 53: 593-601.

Gu H, Zhan W, Ross TJ, Salmeron BJ, Stein EA, Yang Y (eds) (2007). Reduction of functional connectivity in cocaine users revealed by resting-state functional MRI. Proceedings of the
International Society of Magnetic Resonance Medicine. Berlin, Germany.

Haber SN, Knutson B (2010). The reward circuit: linking primate anatomy and human imaging. Neuropsychopharmacology 35: 4-26.

Hanlon CA, Canterberry M, Taylor JJ, DeVries W, Li X, Brown TR et al (2013). Probing the frontostriatal loops involved in executive and limbic processing via interleaved TMS and functional MRI at two prefrontal locations: a pilot study. PLoS One 8: e67917.

Hanlon CA, Dowdle LT, Austelle CW, DeVries W, Mithoefer O, Badran BW et al (2015). What goes up, can come down: novel brain stimulation paradigms may attenuate craving and cravingrelated neural circuitry in substance dependent individuals. Brain Res 1628(Pt A): 199-209.

Hanlon CA, Dufault DL, Wesley MJ, Porrino LJ (2011). Elevated gray and white matter densities in cocaine abstainers compared to current users. Psychopharmacology 218: 681-692.

Hester R, Garavan H (2009). Neural mechanisms underlying drugrelated cue distraction in active cocaine users. Pharmacol Biochem Behav 93: 270-277.

$\mathrm{Hu}$ Y, Salmeron BJ, Gu H, Stein EA, Yang Y (2015). Impaired functional connectivity within and between frontostriatal circuits and its association with compulsive drug use and trait impulsivity in cocaine addiction. JAMA Psychiatry 72: 584-592.

Kelley AE, Berridge KC (2002). The neuroscience of natural rewards: relevance to addictive drugs. J Neurosci 22: 3306-3311.

Koob GF (1996). Drug addiction: the yin and yang of hedonic homeostasis. Neuron 16: 893-896.

Koob GF, Le Moal M (2008a). Addiction and the brain antireward system. Annu Rev Psychology 59: 29-53.

Koob GF, Le Moal M (2008b). Review. Neurobiological mechanisms for opponent motivational processes in addiction. Philos Trans $R$ Soc Lond B Biol Sci 363: 3113-3123.

Kozel FA, Nahas Z, deBrux C, Molloy M, Lorberbaum JP, Bohning D et al (2000). How coil-cortex distance relates to age, motor threshold, and antidepressant response to repetitive transcranial magnetic stimulation. J Neuropsychiatry Clin Neurosci 12: 376-384.

Li SJ, Biswal B, Li Z, Risinger R, Rainey C, Cho JK et al (2000). Cocaine administration decreases functional connectivity in human primary visual and motor cortex as detected by functional MRI. Magn Reson Med 43: 45-51.

Li X, Teneback CC, Nahas Z, Kozel FA, Large C, Cohn J et al (2004). Interleaved transcranial magnetic stimulation/functional MRI confirms that lamotrigine inhibits cortical excitability in healthy young men. Neuropsychopharmacology 29: 1395-1407.

Liang X, He Y, Salmeron BJ, Gu H, Stein EA, Yang Y (2015). Interactions between the salience and default-mode networks are disrupted in cocaine addiction. J Neuroscience 35: 8081-8090.

Liston C, Chen AC, Zebley BD, Drysdale AT, Gordon R, Leuchter B et al (2014). Default mode network mechanisms of transcranial magnetic stimulation in depression. Biol Psychiatry 76: 517-526.

Matochik JA, London ED, Eldreth DA, Cadet JL, Bolla KI (2003). Frontal cortical tissue composition in abstinent cocaine abusers: a magnetic resonance imaging study. Neuroimage 19: 1095-1102.

Middleton FA, Strick PL (2002). Basal-ganglia 'projections' to the prefrontal cortex of the primate. Cereb Cortex 12: 926-935.

Nahas Z, Lomarev M, Roberts DR, Shastri A, Lorberbaum JP, Teneback C et al (2001). Unilateral left prefrontal transcranial magnetic stimulation (TMS) produces intensity-dependent bilateral effects as measured by interleaved BOLD fMRI. Biol Psychiatry 50: 712-720.

Ongur D, Price JL (2000). The organization of networks within the orbital and medial prefrontal cortex of rats, monkeys and humans. Cereb Cortex 10: 206-219.

Politi E, Fauci E, Santoro A, Smeraldi E (2008). Daily sessions of transcranial magnetic stimulation to the left prefrontal cortex gradually reduce cocaine craving. Am J Addict 17: 345-346.

Prisciandaro JJ, Myrick H, Henderson S, McRae-Clark AL, Brady KT (2013). Prospective associations between brain activation to 
cocaine and no-go cues and cocaine relapse. Drug Alcohol Depend 131: $44-49$.

Sheehan DV, Lecrubier Y, Sheehan K, Amorim P, Janavs J, Weiller E et al (1998). The Mini-International Neuropsychiatric Interview (M.I.N.I): the development and validation of a structured diagnostic psychiatric interview for DSM-IV and ICD-10. J Clin Psychiatry 59(Suppl 20): 22-33.

Stefanik MT, Moussawi K, Kupchik YM, Smith KC, Miller RL, Huff ML et al (2013). Optogenetic inhibition of cocaine seeking in rats. Addict Biol 18: 50-53.

Stokes MG, Chambers CD, Gould IC, Henderson TR, Janko NE, Allen NB et al (2005). Simple metric for scaling motor threshold based on scalp-cortex distance: application to studies using transcranial magnetic stimulation. $J$ Neurophysiol 94: $4520-4527$.

Strafella AP, Paus T, Barrett J, Dagher A (2001). Repetitive transcranial magnetic stimulation of the human prefrontal cortex induces dopamine release in the caudate nucleus. J Neurosci 21: RC157.

Terraneo A, Leggio L, Saladini M, Ermani M, Bonci A, Gallimberti L (2016). Transcranial magnetic stimulation of dorsolateral prefrontal cortex reduces cocaine use: a pilot study. Eur Neuropsychopharmacol 26: 37-44.

Tomasi D, Volkow ND, Wang R, Carrillo JH, Maloney T, Alia-Klein N et al (2010). Disrupted functional connectivity with dopaminergic midbrain in cocaine abusers. PLoS One 5: e10815.

Supplementary Information accompanies the paper on the Neuropsychopharmacology website (http://www.nature.com/npp) 